\title{
An improved objective slit-lamp fluorophotometer using tungsten-halogen lamp excitation and synchronous detection
}

\author{
A. TREVOR SMITH, D. P. JONES, G. D. STURROCK, AND PETER WRIGHT \\ From Moorfields Eye Hospital, London
}

SUMmARY A new design of fluorophotometer is described. The instrument can measure lower concentrations of fluorescent dye with greater accuracy than previous models.

Fluorophotometry, in ophthalmology, is the investigation of fluid exchange in the various transparent structures of the eye by the non-traumatic measurement of the rate of change of concentration of an innocuous fluorescent dye (usually sodium fluorescein) that has been introduced into the eye. The fluorescence is excited and observed by means of an optical system which is similar in design to a slitlamp microscope but with provision for measuring the intensity of the fluorescent light collected.

Langham and Wybar $(1953,1954)$ built the first truly objective fluorophotometer which employed a photomultiplier tube (PMT) to measure the intensity of the fluorescent light. Maurice (1963) completely redesigned their instrument, making several important advances. In particular, a clear indication of the position within the eye of the small volume from which fluorescent light was being collected enabled the measurement of fluorescein concentration to be achieved for the first time within structures of the eye other than the relatively bulky aqueous humour. This and Maurice's other new features are incorporated into the present instrument.

Developments since Maurice's instrument have included an attachment designed by Waltman and Kaufman (1970) to allow the conversion of conventional slit lamps into objective fluorophotometers. This attachment, which includes a fibreoptic probe, does not enable discrimination against ambient light to be made. Bloom et al. (1976) have redesigned the electronics of Maurice's instrument and have eliminated any significant instability due to variations in mains frequency by employing synchronous detection. However, they still employ

Address for reprints: P. Wright, FRCS, Moorfields Eye Hospital, City Road, London EC1V 2PD mercury arc excitation, which is inherently unstable, and in practice their instrument is not capable of measuring accurately to as low a concentration as the fluorophotometer described here.

\section{Design}

The layout of the present instrument is shown in Fig. 1. A tungsten-halogen light source driven from a stabilised power supply is used. The optics of the lamp are similar to those of a conventional slit lamp except that the light beam is chopped at $1 \mathrm{kHz}$ by means of a motor-driven disc with 100 slots. The light beam is filtered by a Baird Atomic B4 interference filter $F_{1}$, which passes blue light in the wavelength range $440 \mathrm{~nm}$ to $500 \mathrm{~nm}$. The projected image of the slit $S_{1}$ within the subject's eye is approximately $200 \mu \mathrm{m}$ by $600 \mu \mathrm{m}$.

The monocular microscope gives an upright image for convenience. A portion of the band of fluorescent light which appears in the microscope field of view is selected by means of a window $W$ in the mirror $M_{1}$ which is situated at an intermediate image plane in the microscope and is therefore visible to the operator. Before being allowed to impinge on the PMT photocathode the light so selected is passed through a coloured glass filter $F_{2}$ (Barr and Stroud OG15) which passes visible wavelengths longer than $515 \mathrm{~nm}$ and hence rejects any scattered blue excitation. The projection of the window $\mathrm{W}$ through the microscope objective $\mathrm{O}_{2}$ produces an image approximately $400 \mu \mathrm{m}$ wide and $1,400 \mu \mathrm{m}$ high within the subject's eye which serves to define the region from which fluorescent light can be collected and registered by the detection system.

Since the light exciting the fluorescence is modu- 


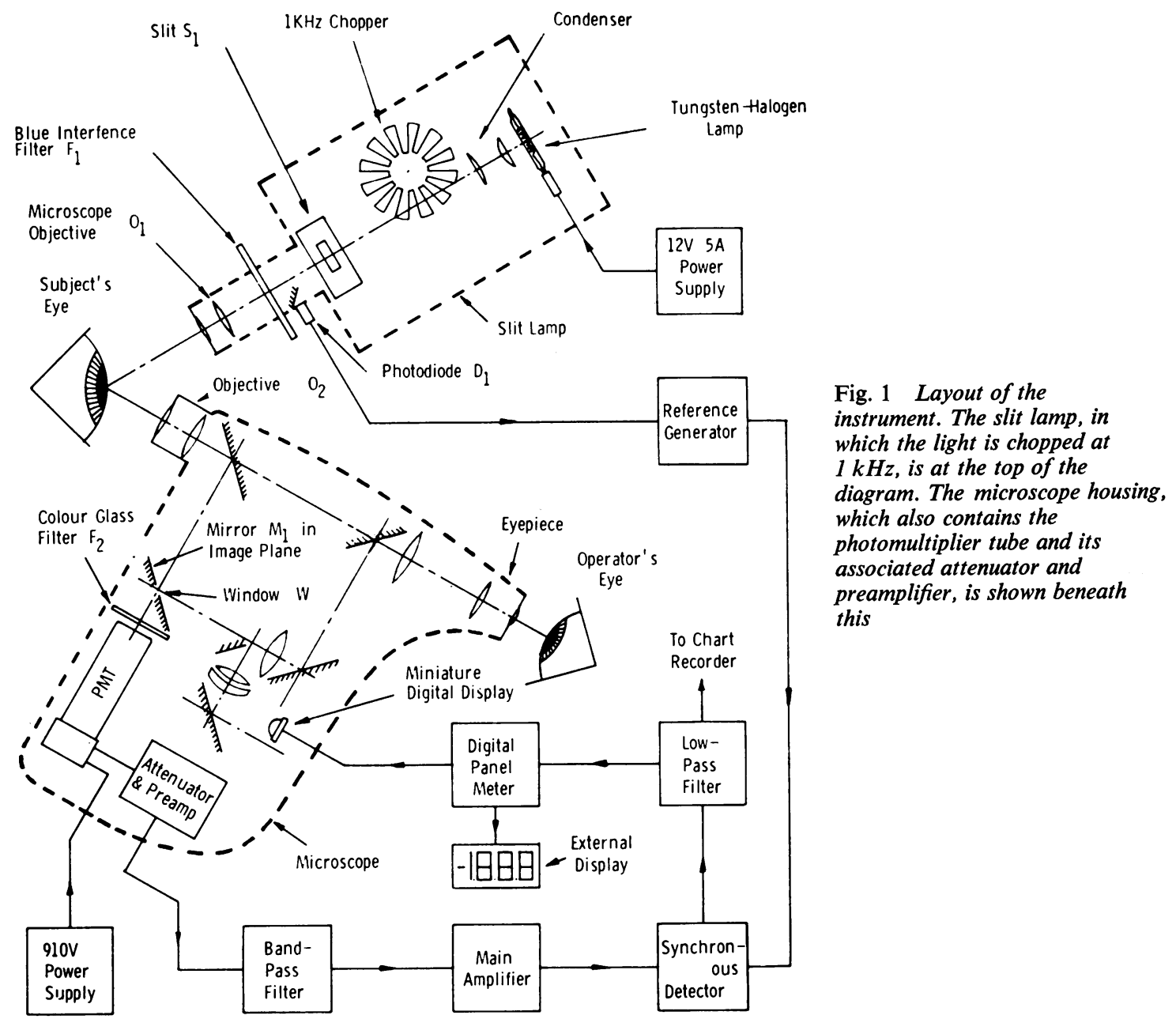

lated, an alternating current signal appears at the PMT anode, thus allowing it to be separated from any direct-current components due to ambient light or PMT dark current. This signal is synchronously detected, the reference being derived from the output of photodiode $D_{1}$, which is illuminated by the chopped incident beam, and then low-pass filtered to reduce fluctuations due to the inherent shot noise from the PMT; provision is also made for three possible filter time constants. The output may either be displayed on a chart recorder or observed on the built-in digital panel meter (DPM). One digital display from this meter is visible in the field of view of the microscope for convenience while a second display is located on the instrument's top panel. Also provided are a preset offset control, which need be adjusted only at the beginning of an experiment, and a control to vary the gain from its normal calibrated value.

\section{Performance}

The linearity of the instrument is shown in Fig. 2. These measurements were obtained by using a series of dilutions of sodium fluorescein in tap water (about pH 8.0) made up the same day in small stoppered test tubes and were achieved without the use of neutral density filters. A single attenuator switch enables accurate measurement of concentrations throughout the linear range from $2 \times 10^{-9} \mathrm{~g} \mathrm{ml}^{-1}$ to $2 \times 10^{-5} \mathrm{~g} \mathrm{ml}^{-1}$, with the results displayed to three significant figures by the DPM. This performance is achieved with the fluorescent light collected from a small volume of height $600 \mu \mathrm{m}$, depth $200 \mu \mathrm{m}$, and 
width $400 \mu \mathrm{m}$, as defined by the intersection of the excitation beam with the projection of the window W through the microscope objective $\mathrm{O}_{2}$.

The use of modern integrated circuit technology, stabilised power supplies, and a tungsten-halogen lamp has resulted in a compact design (Fig. 3), with less than $3 \%$ change in sensitivity for mains voltage variations in the range 200 to $260 \mathrm{~V}$ and less than $2 \%$ of full scale drift measured over 1 hour (after a 20minute warm-up period). The use of a synchronous detection system results in less than $3 \%$ change in sensitivity for a $\pm 8 \%$ change in mains frequency. Careful design has resulted in only $6 \mathrm{~dB}$ of noise being introduced by the photomultiplier, preamplifier, and detection system.

Absolute fluorescein concentrations in the larger transparent media of the eye can be measured by first calibrating the instrument against a standard solution (usually $1 \times 10^{-6} \mathrm{~g} \mathrm{ml}^{-1}$ ) held in a test tube.

\section{Preliminary clinical trial}

Clinical studies have confirmed that the instrument is capable of measuring tear film fluorescein concentration over relatively long periods of time in order to estimate tear flow rate. Moreover, the sensitivity

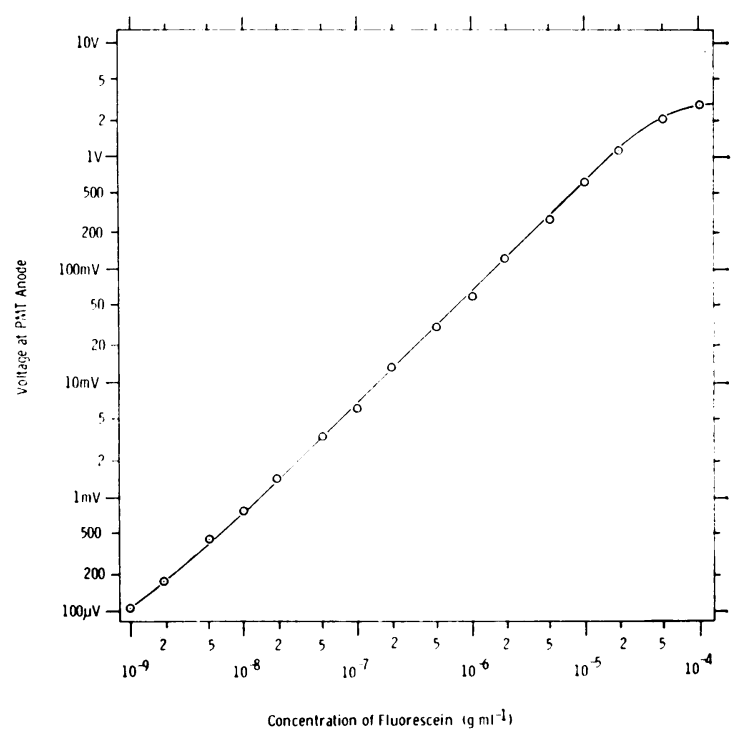

Fig. 2 Response of the instrument to different fluorescein concentrations. This plot of PMT anode voltage versus fluorescein concentration on logarithmic scales was obtained by using successive serial dilutions of sodium fluorescein contained in small stoppered test tubes. The range of practically linear response is from $2 \times 10^{-8} \mathrm{~g} \mathrm{ml}^{-1}$ to $2 \times 10^{-5} \mathrm{~g} \mathrm{ml}^{-1}$

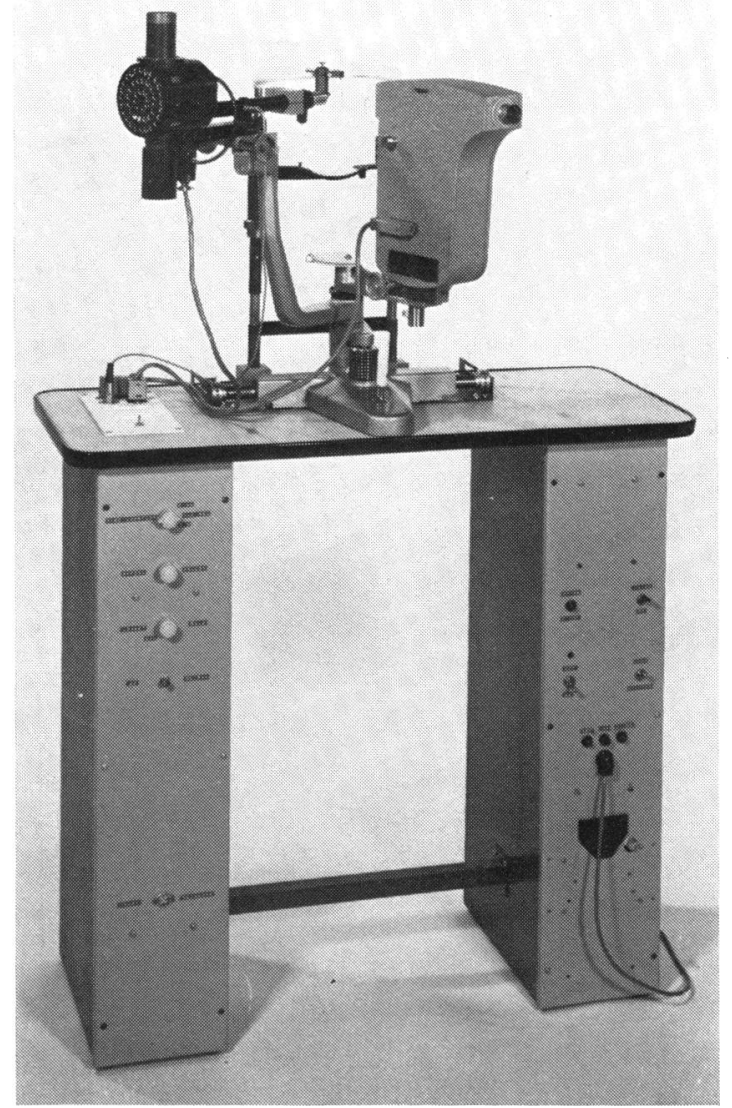

Fig. 3 The complete instrument. The microscope and slit lamp are mounted on the two arms of the slit lamp manipulator. The supporting cabinet contains electronic circuitry for the detection system and the power supplies

range of the instrument is compatible with the measurement of fluorescein concentration not only in the precorneal film but also in the clear ocular media (Maurice, 1967), thus allowing measurement of aqueous flow rate and changes in the diffusion barriers within the eye in a wide range of conditions.

\section{Conclusions}

By using a tungsten-halogen lamp and a synchronous detection system in the design of the present fluorophotometer two sources of instability in previous instruments have been eliminated and the performance has been improved; the whole of the useful range of fluorescein concentrations, from about $2 \times 10^{-9} \mathrm{~g} \mathrm{ml}^{-1}$ to $2 \times 10^{-5} \mathrm{~g} \mathrm{ml}^{-1}$, can be measured without the need for neutral density filters. Furthermore, owing to the extensive use of 
integrated circuits, the amplification and detection system of the final instrument is compact and relatively cheap. In a preliminary clinical trial the instrument was found to be convenient and simple to use for measuring fluorescein concentration in the precorneal tear film.

Dr D. Maurice and Mr W. Buchanan were involved in the early stages of the development of this fluorophotometer, and have given much helpful advice.

\section{References}

Bloom, J. N., et al. (1976). Archives of Ophthalmology, 94, 435.

Langham, M. E., and Wybar, K. C. (1953). Journal of Physiology, 120, 5P.

Langham, M. E., and Wybar, K. C. (1954). British Journal of Ophthalmology, 38, 52 .

Maurice, D. M. (1963). Experimental Eye Research, 2, 33.

Maurice, D. M. (1967). Investigative Ophthalmology, 6, 464.

Waltman, S. R., and Kaufman, H. E. (1970). Investigative Ophthalmology, 9, 247. 